\title{
Le risque de crue en région parisienne Démarche et projets de l'Institution
}

\author{
par J-L Rizzoli \\ Institution Interdépartementale des Barrages-Réservoirs du Bassin de la Seine
}

La protection contre les crues en Région Parisienne résulte de la synergie entre l'action des grands lacs de Seine et la présence de protections locales. Il convient de souligner la complémentarité de ces deux types d'aménagements : les protections locales peuvent protéger contre les inondations faibles et moyennes, les lacs-réservoirs peuvent avoir un impact sur une plus grande gamme de débits.

$\mathrm{Si}$ les grands lacs de Seine ont une contribution reconnue par tous, ils ne contrôlent que $17 \%$ du bassin versant de la Seine à sa confluence avec la Marne, et leurs impacts sont variables suivant le type de crue. De même les protections locales, calées à des niveaux variables en fonction des vallées et des maîtres d'ouvrage concernés, peuvent avoir été limitées par des problèmes d'environnement ou de stabilité.

La région parisienne reste donc vulnérable aux grandes crues.

Les crues du bassin de la Seine en amont de Paris sont lentes, répétitives (généralement de décembre à mars) et relativement prévisibles grâce à leur décalage important par rapport à l'événement pluviométrique qui en est l'origine.

La prévision est donc un outil indispensable pour lutter contre les inondations : il ne devrait théoriquement pas y avoir, en dehors d'accidents, de pertes humaines à redouter, ce type d'inondation ne provoquant que des dommages matériels comme cela a pu être vérifié en 1910.

La réduction de ces dommages passe alors par des mesures de prévention et des mesures de protection, qui nécessitent des études économiques pour en apprécier l'intérêt.
Ces constats ont conduit l'Institution, pour poursuivre sa mission liée aux crues, à accepter de prendre la maîtrise d'ouvrage des études qui devront lui permettre :

* de se fixer des objectifs de protection contre les crues en Région Parisienne,

* de poursuivre ses recherches pour améliorer la gestion des ouvrages existants,

* de préparer d'éventuelles décisions futures d'investissements nouveaux.

Ainsi c'est principalement dans un cadre de "préventionprotection-information" que se situe la démarche de l'Institution en liaison avec l'ensemble des acteurs concernés par les crues et notamment ceux qui sont chargés de l'annonce des crues.

\section{I — LA DÉMARCHE}

Afin d'apprécier l'intérêt d'éventuelles nouvelles opérations de protection contre les crues, l'Institution s'est portée maitre d'ouvrage d'une étude relative à l'évaluation des enjeux socio-économiques des crues de la Seine et de la Marne en région Ile-de-France.

Une telle étude doit permettre de mieux appréhender l'impact économique d'une crue centennale analogue à la crue de 1910 ou de toute autre crue, de quantifier "l'efficacité hydraulique et économique" des ouvrages existants, de mieux évaluer les risques subsistants malgré les lacs-réservoirs et d'établir une hiérarchisation des aménagements futurs suivant un critère exprimé en terme de coût/avantage.

In spite of the presence of various local flood protections and the attenuating function of the great lakes, the Paris area remains vulnerable to damage in case of a major flood. For this reason, the IIBR - the Interdepartmental Institution for the Dams and Reservoirs of the Seine River Basin (Institution Interdépartementale des Barrages-Réservoirs) as part of its mission, has become a Prime Contractor for risk evaluation studies. These studies will investigate the risks involved when rivers are in flood and will test the technical feasibility of potential future construction work, thus addressing the concerns of local government authorities, residents and representatives of the economic sector. The IIBR's approach, which operates within a broader "prevention-protection-information" framework, and the various projects under consideration to reduce flood damage, are described in this presentation. 
Un programme général d'études a été mis au point en 1990, par l'Institution en collaboration avec les Services de l'Agence de l'Eau Seine-Normandie, la Région Ile-de-France, la Direction Régionale de l'Equipement d'Ile-de-France, la Délégation aux Risques Majeurs du Ministère de l'Environnement, la Direction Régionale de l'Environnement d'Ile-deFrance, le Service de la Navigation de la Seine, l'Institut d'Aménagement et d'Urbanisme de la Région Ile-de-France (IAURIF) et le Centre d'Enseignement et de Recherche pour la Gestion des Ressources Naturelles et de l'Environnement (CERGRENE), tous intéressés par les divers aspects que peuvent présenter les dommages issus des crues.

\subsection{Première phase de l'étude économique}

Compte tenu du caractère méthodologique expérimental que présente cette étude, une réflexion plus approfondie a conduit à définir une première phase dont l'objet a été d'effectuer une investigation prospective, par un traitement de l'information existante, pour déterminer plus précisément les limites de ce qu'il était raisonnablement possible d'entreprendre, soit :

- d'identifier les sources d'informations, puis collecter les données disponibles sur les désordres hydrauliques et les dommages pouvant en résulter en zone urbanisée d'Ile-deFrance. Ces informations ont été structurées, afin de faire apparaître une hiérarchisation des désordres et des dommages, en fonction des niveaux de submersion ;

- de réaliser un modèle numérique de terrain permettant la cartographie des crues et, par utilisation des fichiers du "Mode d'occupation des sols" (MOS) de l'Institut d'aménagement et d'urbanisme de la Région Ile-de-France, de réaliser les calculs de dommages ;

- d'établir, à partir des données disponibles, une première évaluation des dommages résultant du niveau des crues caractéristiques observées dans le passé (1910 - 1955 1970).

L'aire de l'étude de la première phase a été délimitée à l'amont par les agglomérations de Melun sur la Seine et de Meaux sur la Marne, à l'aval par celle de Mantes-la-Jolie et sur les affluents par la limite de la zone d'influence des crues de la Seine et de la Marne.

Les lignes d'eau des crues historiques ont été considérées comme identiques à ce qu'elles étaient à l'époque où elles sont intervenues et elles ont été reportées sur l'état actuel de la topographie et de l'urbanisation.

Les bases de données ainsi constituées regroupent les informations relatives à la zone inondée, la hauteur d'eau et la durée de submersion.

Les désordres potentiels ont été localisés et caractérisés, dans la limite des données disponibles après enquêtes, et constitués en base de donnée.

La démarche adoptée pour estimer les dommages directs a consisté à collecter et analyser les données sur la zone d'étude afin d'obtenir une caractéristique de chaque type d'occupation des sols en nature et en valeur modulées par des coefficients représentant la diversité de l'occupation des sols au niveau communal et des taux d'endommagement principalement dimensionnés par la hauteur de submersion.

Deux types de dommages indirects ont été estimés dans cette étude : les dommages indirects définissables au travers des zones MOS (coût de nettoyage, de relogement, des services d'intervention, des pertes d'exploitation, des pertes de valeurs d'usage, ...) et les dommages indirects sur les réseaux
(AEP, EDF, GDF, PTT,...) et les transports qui ont été traités de façon globale sur l'ensemble de la région.

La première phase de cette étude a permis de se faire une idée assez précise des difficultés humaines, économiques et sociales qui se présenteraient en cas d'une inondation qui aurait la même ligne d'eau que 1910,1955 et 1970 dans les conditions économiques actuelles sans l'action des lacs-réservoirs. Les résultats (tableau 1) permettent de souligner l'importance des protections locales et de dégager les ordres de grandeur des différents dommages directs et indirects dus aux crues. En particulier, le coût d'une inondation "centennale" est très important : il est évalué à 53 milliards de francs. Il a été multiplié par 6 environ (en valeur actuelle) depuis 1910 selon les estimations que nous connaissons de cette catastrophe. La vulnérabilité de la région parisienne a donc été considérablement augmentée du fait de son urbanisation.

\subsection{Prévention - information}

Les résultats de la première phase conduisent à mettre l'accent sur le fait que la réalisation d'éventuelles protections supplémentaires doit avoir comme objectif la protection de l'existant et non pas être le prétexte à une urbanisation ayant pour conséquence l'augmentation de la vulnérabilité de la région. C'est pourquoi il faut souligner l'importance du volet prévention qui comprend notamment la cartographie des risques inondation et l'information préventive. C'est dans ce cadre qu'une large diffusion de cette étude a été réalisée : elle vise les administrations concernées (Mairie, Préfectures, Départements) et les organismes ayant offert des éléments techniques, dans le double objectif :

- de permettre une prise de conscience des problèmes conduisant à d'éventuelles actions en vue de diminuer le coût des dommages ;

- par retour d'information de pouvoir alimenter les bases de données pour la réalisation des phases ultérieures permettant d'améliorer l'appréciation des dommages et des désordres.

\begin{tabular}{|l|c|c|c|}
\hline & 1910 & 1955 & 1970 \\
\hline Dommages directs : & & & \\
Logement & 14,4 & 3,5 & 1,2 \\
Activités & 13,4 & 4,4 & 2,1 \\
Equipements et divers & 5,5 & 2,0 & 0,6 \\
TOTAL & 33,3 & 9,9 & 3,9 \\
\hline Dommages indirects : & & & \\
Pertes d'exploitation et divers & 8 & 2,1 & 1,9 \\
Intervention des services de & 0,25 & 0,1 & 0,05 \\
Secours & & & \\
Electricité & 4,2 & 2,5 & 1,00 \\
Distribution de l'eau & 0,55 & & \\
Transports routiers & 2 & 0,2 & 0,05 \\
Métro & 3,15 & & \\
RER & 0,45 & & \\
SNCF & 0,5 & 0,3 & \\
TOTAL & 19,1 & 5,2 & 3 \\
\hline TOTAL & $\mathbf{5 2 , 4}$ & $\mathbf{1 5 , 1}$ & $\mathbf{6 , 9}$ \\
\hline
\end{tabular}

Tableau 1. - Coûts des dommages dus à une crue type sans l'action des barrages-réservoirs (en milliards de francs) de la Seine entre Melun (77) et Mantes (78) et de la Marne à l'aval de Meaux (77). 


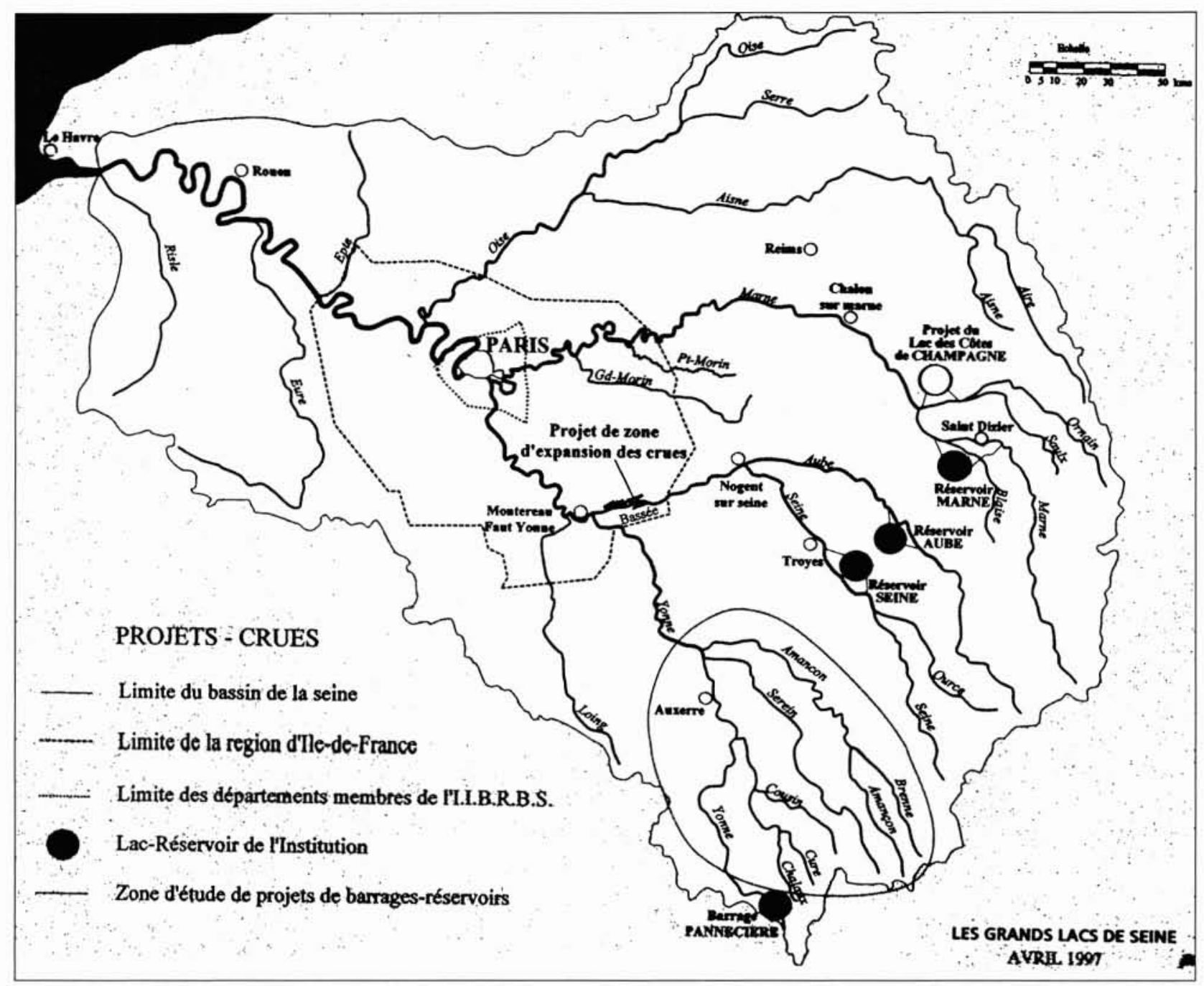

1. Etat actuel et futur des grands lacs-réservoirs.

A titre d'exemple, suite à la diffusion de ces rapports, divers services publics ont commencé à établir des plans de prévention et de protection : France Télécom a cherché à affiner la connaissance de la vulnérabilité de certains centraux téléphoniques, la RATP envisagerait de rehausser $($ de $70 \mathrm{~cm}$ ) le niveau de protection de ses installations afin de le caler sur celui de la crue de 1910, EDF incorpore ce risque dans l'évolution de son réseau de distribution, GDF examine les solutions permettant de protéger ses postes de détente, etc ...

\subsection{Poursuite de l'étude économique : l'outil}

Les prélèvements opérés par les lacs-réservoirs se traduisent par une limitation des débits et donc des hauteurs d'eau à l'aval de ces ouvrages. Le modèle MSBR permet de simuler l'impact de ces lacs sur les débits de la Seine et de la Marne à l'entrée de l'agglomération parisienne. Mais les résultats en débit ne permettent pas de définir quelles seraient les lignes d'eau correspondantes sur les axes Seine amont, Marne, Seine aval.

La seconde phase de l'étude que l'Institution vient d'engager avec ses partenaires doit permettre de quantifier l'efficacité hydraulique et économique des ouvrages de protection existants et futurs grâce à la réalisation d'un nouveau modèle hydraulique qui pourra établir les différentes lignes d'eau correspondant aux débits simulés en région Ile-de-France.
Les résultats de cette deuxième phase interviendront fin 1997.

Cette étude fournira ainsi un outil permettant de définir des objectifs cohérents de protection contre les crues de la Région Parisienne et d'avoir une vision globale dans la mise en cuvre des moyens capables de les atteindre.

\subsection{Projets et Concertation}

La démarche de l'Institution intègre le recensement de tous les projets et solutions techniques envisagés pour réduire la vulnérabilité de la Seine et de ses affluents face aux crues (Figure 1).

Le principe de tous les ouvrages décrits ci-après est inscrit dans le Schéma Directeur d'Aménagement et de Gestion des Eaux (SDAGE) Seine-Normandie.

Pour tous ces projets éventuels, l'Institution engagera le moment venu des concertations directes avec les responsables politiques, économiques, associatifs, administratifs des sites concernés par ses projets : c'est ce qui a été réalisé pour le projet du lac des Côtes de Champagne. Leur participation est en effet nécessaire :

— pour réaliser les études techniques, écologiques, économiques, sociales, etc.... préalables à la définition plus précise des projets : 
— pour évaluer le coût des ouvrages ;

- pour estimer le coût des mesures d'accompagnement qui risquent de prendre à l'avenir une proportion de plus en plus importante.

\section{II — LES PROJETS}

\section{- 2.1 Amélioration de la gestion des lacs-réservoirs actuels}

L'exploitation des lacs-réservoirs est réalisée conformément à des règlements d'eau élaborés au moment de leur construction, et donc établis en fonction des connaissances hydrologiques du moment.

Depuis, des améliorations ponctuelles ont été réalisées à l'intérieur des libertés permises par ces règlements d'eau et ce en concertation avec les partenaires de l'Institution dont le Ministère de l'Environnement et ses directions régionales, l'Agence de l'Eau, la Région Ile-de-France, des scientifiques du PIREN SEINE etc.

La réflexion concernant l'amélioration s'articule autour des thèmes suivants :

- Amélioration locale de la gestion,

- Prévisions des débits à l'amont des lacs-réservoirs.

A partir de la mise en place d'un système d'acquisition de données hydrométéorologiques en temps réel installé dans le bassin amont, il sera possible début 1998 de prévoir les débits à l'amont de chaque ouvrage aussi bien en période de crues (d'hiver et de printemps) qu'en période d'étiage.

- Des bilans hydrologiques au droit de chaque ouvrage sont en cours pour évaluer les imprécisions des dispositifs de mesure, proposer des améliorations et suivre leur évolution dans le temps. A titre d'exemple, de nouvelles courbes hauteur-volume sont établies dans le cadre des vidanges décennales (Pannecière en 1991, Marne en 1993, Aube en 1995, Seine en 1997).

- Connaissance de l'impact des lacs-réservoirs sur l'environnement.

- Une gestion globale des ouvrages au niveau du bassin.

- L'Institution avec l'outil MSBR, correspondant à un modèle de propagation des crues, peut réaliser une certaine prévision de celles-ci lors de leur suivi en temps réel.

- Pour faire fonctionner cet outil et améliorer aussi la gestion de ses ouvrages, l'Institution a besoin d'une acquisition en temps réel de données issue de stations limnimétriques situées dans le bassin de la Seine à l'aval de ses ouvrages. Elle participe en conséquence à la réorganisation et à la modernisation du réseau hydrométrique du bassin de la Seine. Toutes ces installations pourraient constituer à terme les composantes d'un centre de gestion des eaux du bassin de la Seine à l'amont de Paris.

Si l'amélioration de la gestion des lacs-réservoirs est nécessaire, elle aura néanmoins ses limites. Il y a donc lieu d'examiner toutes les variantes possibles permettant de faire face aux crues : les sites possibles pour des grands barrages ont été recensés mais d'autres aménagements ont été imaginés (protections locales, tunnel évacuateur de crues court-circuitant une boucle de la Seine en aval de Paris, l'aménagement de zones naturelles d'expansion de crue, etc.).

\subsection{Barrages-réservoirs dans le Bassin de l'Yonne}

L'analyse des crues historiques sur le Bassin de la Seine montre que la pointe de crue de la Seine à Paris correspond au passage de la pointe de crue du Bassin de l'Yonne, tout au moins pour les épisodes où cette rivière participe fortement à la crue. La participation de l'Yonne à la pointe de la crue à Paris est généralement supérieure à $40 \%$.

L'étude de projets d'ouvrages dans I'Yonne a été entreprise dès le début de ce siècle et de nombreux sites ont été reconnus mais pas équipés. Ce constat tient à la difficulté intrinsèque du problème : les sites favorables à la construction du réservoir sont situés dans le Morvan mais ne contrôlent qu'une part modeste du bassin versant. De plus, seul l'équipement de chacune des rivières principales par un ouvrage régulateur peut assurer une action significative sur la genèse de la crue.

Dans la partie aval du bassin versant, les sites sont rares et posent des difficultés de réalisation, liées à la géologie (auréole jurassique et crétacé donnant des cuvettes perméables), à la topographie défavorable et surtout à l'occupation des vallées principales qui conduit à rechercher les sites en dérivation sur des vallées adjacentes.

C'est pourquoi l'Institution a réorienté son approche, d'une part en privilégiant la fonction écrêtement de crue sur la fonction de soutien des étiages (un ouvrage géré en pur écrêteur de crue permettrait ainsi de rechercher des sites plus modestes que ceux pris en compte dans les études antérieures), d'autre part, afin de limiter les impacts écologiques de tels ouvrages, il pourrait être envisagé des ouvrages à "pertuis ouverts", sur le cours même de la rivière, sans retenue permanente (laissant passer l'eau, sauf en crue) et/ou la surélévation des barrages existants.

L'étude montre qu'il est possible, en réalisant plusieurs ouvrages judicieusement combinés, pour un coût total de l'ordre de 2200 millions de francs, de réduire le maximum de crue d'environ 50 à 60 centimètres en région parisienne. Des incertitudes subsistent cependant sur l'impact de ces lacs et sur leurs modes de gestion qui restent donc à approfondir.

\subsection{Utilisation optimale de la zone de la Bassée en Seine et Marne}

La Bassée correspond à un élargissement du lit majeur de la Seine, situé à $80 \mathrm{~km}$ en amont de Paris. Cette "cuvette" dans laquelle serpentent la Seine et de nombreux petits affluents, occupe une superficie de 10000 ha $\left(100 \mathrm{~km}^{2}\right)$ entre Nogent-sur-Seine, Bray et Montereau. Elle constitue :

- la plus grande plaine inondable du bassin : c'est une zone naturelle d'expansion des crues de la Seine, dont elle atténue les effets en aval,

- une zone humide : la plus importante de la région Ile-deFrance,

- une grande richesse floristique et faunistique,

— un aquifere important pour la région.

dont le caractère a été modifié par :

— l'exploitation des sables et graviers alluvionnaires,

- les travaux de recalibrage du lit mineur pour la navigation qui augmentent la vitesse des crues, aggravent le risque d'inondation à l'aval et suppriment tout débordement.

A l'amont de Bray, la Seine à une capacité de transit inférieure à $200-250 \mathrm{~m}^{3} / \mathrm{s}$ qui la fait déborder régulièrement. A l'aval, sa mise à grand gabarit a porté sa capacité à $400 \mathrm{~m}^{3} / \mathrm{s}$ et lui permet d'écouler la totalité des débits sans débordement, le débit de pointe ne dépassant généralement pas $350 \mathrm{~m}^{3} / \mathrm{s}$ par action des barrages Seine et Aube. La zone entre Bray et Montereau, qui était initialement inondable, ne l'est pratiquement plus. 
L'idée de base du projet consiste donc à remplir une partie du lit majeur de la Seine dans la Bassée entre Bray et Montereau au moment du passage de la pointe de crue de l'Yonne à Montereau de façon à ce que le creux de débit créé sur la Petite Seine permette de diminuer le débit de pointe à l'aval de la confluence avec l'Yonne.

Ainsi, dans ce secteur, il pourrait être conçu un aménagement relativement conséquent de type Polder (comme ceux réalisés le long du Rhin) pouvant stocker environ $45 \mathrm{Mm}^{3}$ et permettre à cette partie de la Bassée de retrouver son caractère de zone humide tout en ayant un impact sur les grandes crues en région Ile-de-France et en préservant les activités et les lieux habités de la zone concernée.

L'utilisation de la Bassée, comme zone d'expansion contrôlée des crues nécessite donc des études et des négociations complexes. Elle pourrait, néanmoins, être un projet fédérateur qui inclurait les autres contraintes, et en particulier l'aspect écologique : les inondations qui ont disparu dans certains secteurs pourraient y être réintroduites de façon limitée, chaque année. pour reconstituer des zones humides.

L'Institution a engagé une réflexion dans ce sens et a fait étudier en parallèle l'effet d'un tel aménagement sur les inondations en région parisienne.

Il s'agirait d'y réaliser sept casiers sur une superficie totale de 2400 hectares, délimités par des digues de 2 à 4 mètres de haut, dans lesquels serait pompée une partie de l'eau des crues de la Seine. Le système fonctionnerait tous les 5 - 6 ans pendant une semaine environ.

L'étude, qui est commune à celle des réservoirs dans le bassin de l'Yonne montre que le gain en hauteur est de l'ordre de 30 à 35 centimètres en région parisienne pour un coût évalué à $1500 \mathrm{MF}$ T.T.C. Ce projet est réalisable de façon plus progressive qu'un lac-réservoir, car il peut être construit casier par casier.

\subsection{Le Lac des Côtes de Champagne}

Reprenant un projet élaboré par les services de l'Etat et I'Agence de l'Eau dans les années 1980. I'Institution a engagé, dès 1992, une concertation locale avec les élus, les responsables économiques et associatifs, et les administrations du département de la Marne, concernés par le projet du Lac des Côtes de Champagne.

Elle a abouti en mars 1996 à un avant-projet dont les caractéristiques et les objectifs ont pris en compte les besoins et suggestions formulées par les membres des groupes de travail.

C'est un projet, situé sur le dernier grand site potentiel d'implantation d'un lac-réservoir en Champagne, dont les principes généraux de construction et d'aspects sont ceux des lacs-réservoirs Seine, Marne et Aube : un ouvrage en dérivation dont les digues en terre ne barrent pas la vallée principale.

- Le réservoir créé, d'une surface d'environ 2600 hectares, et d'une capacité totale d'environ $150 \mathrm{Mm}^{3}$ serait délimité au sud par deux digues de 15 à $18 \mathrm{~m}$ de hauteur et d'une longueur totale de $8 \mathrm{~km}$ environ. Des digues internes seraient réalisées afin de conserver des plans d'eau permanents aux abords des villages qui ne seront ainsi pas touchés et de délimiter des bassins à vocation touristique et écologique.

Le remplissage de la retenue serait assuré par les apports de sous-affluents de la Marne (Ornain, Chée, Vière). La restitution se ferait dans la Saulx, par un canal parallèle à l'écoulement des eaux, à flanc de coteau dans la vallée, prolongé par un tunnel jusqu'à la rivière.
- Le rôle du lac des Côtes de Champagne dont le coût est estimé à 1500 M.F T.T.C serait double : soutien des étiages et écrêtement des crues.

Ainsi il pourrait être dégagé une tranche d'écrêtement des crues allant jusqu'à $130 \mathrm{Mm}^{3}$ et une tranche de soutien d'étiage d'environ $118 \mathrm{Mm}^{3}$.

Le projet initial concernait principalement le soutien des étiages de la Marne et de la Seine aval, rivières particulièrement fragiles en cas de sécheresse. C'est pourquoi dans le cadre des groupes de travail, on n'a retenu une tranche d'écrêtement de crue que d'environ $60 \mathrm{Mm}^{3}$ pour réduire les dommages dus aux seules inondations locales. Il s'avère cependant que ce stockage, opéré lors des crues, permettrait de diminuer la hauteur des inondations de $30 \mathrm{~cm}$ le long de la Marne en région parisienne et de $10 \mathrm{~cm}$ le long de la Seine à l'aval de la confluence avec la Marne.

Compte tenu de l'intérêt de ces premiers résultats, dans le cadre de l'élaboration ultérieure du projet technique, il serait intéressant de voir ce qu'apporterait l'augmentation de la tranche de crue jusqu'à $130 \mathrm{Mm}^{3}$. Les études devront peaufiner cet aspect qui permettrait au Lac des Côtes de Champagne d'augmenter sa contribution à la réduction des coûts des dommages dus aux crues de la Marne et de la Seine aval en région Ile-de-France. Ces gains pourraient encore être valorisés par une meilleure gestion coordonnée avec le lac réservoir Marne.

\subsection{Tunnel de dérivation de la boucle de Gennevilliers}

Ce projet correspond a celui que la Direction Régionale de l'Equipement d'lle-de-France avait étudié à partir de 1985-86.

Il s'agirait de diminuer la hauteur des inondations dans la partie centrale de la région parisienne, en faisant passer un débit de $600 \mathrm{~m} 3 / \mathrm{s}$, prélevé pendant les crues de la Seine, dans un tunnel, dont l'entrée serait à Issy-les-Moulineaux et la sortie serait située à la limite des départements des Hauts-deSeine et des Yvelines aux environs de Port Marly et du Pecq. Ce tunnel aurait $10 \mathrm{~m}$ de diamètre et $13 \mathrm{~km}$ de long environ.

Les débits de la Seine qui commencent à produire des perturbations en région parisienne sont de $900 \mathrm{~m}^{3} / \mathrm{s}$ à Paris (coupure des voies sur berges) puis de $1230 \mathrm{~m}^{3} / \mathrm{s}$ (plus hautes eaux navigables). Dans la gamme de débits 900 $2400 \mathrm{~m}^{3} / \mathrm{s}$ (crue de 1910), un prélèvement jusqu'à $600 \mathrm{~m}^{3} / \mathrm{s}$ (il serait possible de faire passer par gravité jusqu'à $300 \mathrm{~m}^{3} / \mathrm{s}$ et de compléter par pompage) permettrait de diminuer le débit de crue dans la boucle de Gennevilliers et la hauteur des inondations de $1.2 \mathrm{~m}$ à $0,2 \mathrm{~m}$ au fur et à mesure qu'on s'éloigne vers l'aval de la prise d'eau. A l'amont dans Paris, et dans une moindre mesure dans le Val-de-Marne le long de la Seine et de la Marne et en Seine-Saint-Denis le long de la Marne, "l'aspiration" dans le tunnel permettrait de diminuer, à débit constant, la hauteur de la ligne d'eau (de $1,1 \mathrm{~m}$ à 10 $\mathrm{cm})$. Ces effets seraient obtenus sans pour autant renforcer significativement la crue en aval de la restitution de l'ouvrage.

Ce projet pourrait intéresser également le Syndicat Intercommunal de l'Assainissement de l'Agglomération Parisienne (SIAAP), qui utiliserait le tunnel pour stocker au printemps et en été des premières eaux d'orage provenant de collecteurs locaux. évitant ainsi leur reversement dans la Seine (cause de mortalités piscicoles et de dégradation de la qualité), avant de les renvoyer progressivement vers ses stations d'épuration.

Les diverses études menées par l'Institution et le SIAAP. montrent que des difficultés techniques sont à résoudre : 
insertion de l'ouvrage de prise et de la station de pompage, mesure exacte de l'impact du flux sortant du tunnel sur les inondations à l'aval etc. Le coût total de l'opération serait de l'ordre de 5 milliards de francs T.T.C. Compte tenu de son coût et des difficultés à résoudre, ce projet est provisoirement écarté par l'Institution.

\subsection{Ouvrages locaux de protection}

Il s'agit principalement des digues et murettes qui peuvent être mises en cuvre progressivement pour protéger des zones urbanisées de l'envahissement des eaux. Il ne s'agit pas évidemment de réaliser des endiguements à l'amont de l'agglomération parisienne qui supprimeraient les zones d'expansion des crues, véritables réservoirs naturels, qu'il convient de préserver, voire de valoriser.

Il est cependant à noter que ces protections locales peuvent ne pas toujours être bénéfiques :

- Ces endiguements, s'ils ont des conséquences favorables localement, peuvent avoir des effets néfastes à l'amont et surtout à l'aval des zones protégées.

- La présence des protections, en créant un pseudo climat de sécurité peut aggraver les dommages en cas de défaillance (rupture par manque d'entretien par exemple) ou en cas de crue très exceptionnelle (dépassement du seuil de protection) : les zones ainsi protégées restent fragiles.

Un inventaire réalisé en 1983 pour le compte de la Région Ile-de-France avait fait apparaitre qu'on pouvait réaliser dans les quatre départements constitutifs de l'Institution pour 315 MF (en Francs 95) de travaux neufs de protection prioritaires, pour $405 \mathrm{MF}$ de travaux neufs complémentaires et pour $420 \mathrm{MF}$ de rénovation d'ouvrages, soit un total de plus de $1100 \mathrm{MF}$, hors coût d'entretien.

Des aménagements ont été réalisés depuis cette date. La deuxième phase de l'étude sur le coût des dommages dus aux inondations engagée par l'Institution actualisera toutes les données sur ce type de projet et établira les avantages en termes de réduction de dommages. Mais surtout l'outil issu de cette étude permettra :

- d'appréhender l'impact global et cumulatif de la mise en œuvre de protections localisées et donc de prendre avec discernement la décision de les réaliser ou non,

- de simuler la rupture d'une protection et d'appréhender par exemple les enjeux socio-économiques résultant d'un mauvais entretien de ces protections,

- et ainsi de se fixer des priorités dans leur mise en auvre et leur entretien. 